[With the increasing public interest in astronomy, professional astronomical institutions have devised many ways of handling enquiries and also carrying out general public education. Specific details of some of the methods used were given in four contributions. - Eds.]

\title{
Feeding the Five Thousand
}

\section{Muriel Enock}

1230 Verdier Avenue 102, Brentwood Bay,

British Columbia, Canada VOS 1A0

The Dominion Astrophysical arranged 20 special viewing sessions for Comet Halley, and although most of these were clouded out, some 3000 people attended. The Observatory receives 30000 visitors annually and its display area was recently renewed. This was done on a very limited budget, but the display succeeded in providing both scale and distance models of Solar-System bodies, telescope models, and much visual material, all accompanied by bi-lingual text.

\section{Efforts by the Instituto de Astrophisica de Canarias to Publicise Astronomy}

Ignacio Garcia de la Rosa, JI. Cepa, M.R. Kidger, A. Mampaso, L. Martinez, F. del Puerto, C. Sanchez, Ll. Tomas

Instituto de Astrophisica de Canarias, E-38200 La Laguna (Tenerife), Spain

The Institute provides facilities for school and group visits and these have also included "Astronomical Weeks" (held on more than one of the islands in the group) and yearly open days. In 1985 the formal opening of the Institute engendered so much publicity that at the first open day at the Rocque de los Muchachos Observatory, 15000 people attended. (The island's total population is 80000 .) During 1986 , a special event organized at a beach on Tenerife was attended by an estimated 70000 people, thousands more being prevented by an enormous traffic jam. A month later, the inhabitants of La Palma switched off all private and street lights throughout the island for a period of 3 hours. Because of the interest and demand from local people and tourists, additional visitors' centres are planned.

\section{Tailor-Made Messages:}

\section{Astronomical Information for Diverse Audiences}

James Cornell

Smithsonian Astrophysical Observatory, Cambridge, MA 02138, U.S.A.

The SAO offers a wide range of public-relations services. For example, research results may be transmitted, via reporters, to newspapers or television stations. More 